\title{
Uma Experiência de Implantação de Processo de Testes - Caso PRODEPA
}

\author{
Renata W. Monteiro ${ }^{1}$, Amanda Sizo ${ }^{1}$, Tácio Vinicius ${ }^{1}$, Luis Felipe Guimaràes ${ }^{1}$, \\ Cláudio Roberto de Lima Martins ${ }^{1}$, Ernani Sales ${ }^{2}$, Carla A. Lima Reis ${ }^{2}$ \\ ${ }^{1}$ Diretoria de Desenvolvimento de Sistemas - Empresa de Processamento de Dados do \\ Estado do Pará (PRODEPA) - Belém - PA - Brasil \\ ${ }^{2}$ Laboratório de Engenharia de Software - Universidade Federal do Pará (UFPA) - \\ Belém - PA - Brasil \\ \{renata.monteiro, amanda.sizo,tacio.ribeiro, luis.guimaraes, \\ claudio.martins\}@prodepa.pa.gov.br, ernani@webapsee.com, clima@ufpa.br
}

\begin{abstract}
This paper reports a testing process definition and deployment experience at PRODEPA. In this report, we present the main results obtained with this experience: the PRODEPA Testing Process definition; the learned lessons and an initial quantitative analysis. Currently, there are two projects enacting this testing process and the preliminary results show an evidence of significant improvement on the developed software products quality.
\end{abstract}

Resumo. Este artigo relata a experiência de definição e implantação de um processo de testes na PRODEPA. Nesse relato são apresentados os principais resultados dessa experiência: o processo de testes da PRODEPA (em termos de atividades, artefatos, papéis, ferramentas, entre outros componentes que viabilizam sua execução), lições aprendidas e uma análise quantitativa inicial. Atualmente, dois projetos estão executando o processo de testes e os resultados preliminares apontam para um significativo ganho na qualidade do produto final.

\section{Introdução}

Segundo BOEHM e BASILI (2001), 40\% a 50\% do esforço de um projeto de desenvolvimento de software é gasto com retrabalho que poderia ser evitado. Para minimizar esse problema o software é testado para descobrir erros que foram feitos inadvertidamente no momento em que foi projetado e construído [Pressman 2006].

Um processo de testes de software pode ser definido como um conjunto de passos ordenados constituídos por atividades, métodos e práticas, usadas para testar um produto de software [Crespo et al. 2008]. Sendo assim, um processo de teste maduro e bem definido auxilia na construção de softwares eficientes e agrega qualidade ao produto final da empresa.

Entretanto, apesar da adoção de um processo sistematizado de testes ser de fundamental importância para o crescimento de inúmeras empresas de desenvolvimento de software, pois através de procedimentos eficientes de testes durante todo $\mathrm{o}$ desenvolvimento do produto se garante a redução do número de defeitos, pode haver possíveis problemas na aplicação desse processo, tais como: a não utilização das devidas técnicas, a falta de preparo da equipe de teste, entre outros; que devem ser detectados precocemente para que não resulte num processo de testes descontrolado e ineficiente. 
Nesse sentido, a PRODEPA cujo objetivo é "ser referência na gestão de Tecnologia da Informação e Comunicação no Estado do Pará, através da excelência na prestação de serviços e da proposição de soluções inovadoras", vem buscando aperfeiçoar seus métodos e práticas de desenvolvimento para que possa alcançar melhores resultados e atender seu cliente com uma maior qualidade.

Tais iniciativas estão alinhadas com o programa de melhoria contínua implantado na empresa, que foi avaliada nível F do MPS.BR [MPS.BR 2010] em 2007 e atualmente vem agregando ao seu processo padrão outros processos que, do ponto de vista da empresa, são de suma relevância para a evolução do trabalho, assim como para a excelência de seus produtos.

Nesse relato serão apresentados os principais pontos resultantes da experiência de definição e implantação do processo de testes da PRODEPA: o processo de testes, definido em parceria com a Universidade Federal do Pará (UFPA); as lições aprendidas, coletadas em entrevistas com os participantes do processo; e uma análise quantitativa inicial, resultado da medição da taxa de defeitos dos sistemas desenvolvidos antes e depois da implantação do processo.

Este artigo está organizado em cinco seções, além desta introdução. A seção 2 descreve como o processo de testes foi implantado na PRODEPA bem como detalha, na seção 3 é feita uma analise quantitativa do processo e seus resultados, na seção 4 são relatadas as lições aprendidas e finalmente a seção 5 apresenta as conclusões desta experiência.

\section{Definição e Implantação do processo de testes da PRODEPA}

Em março de 2008 foi iniciado um projeto de parceria entre PRODEPA e UFPA a partir de um acordo de cooperação técnico-científica apoiado pela Secretaria de Desenvolvimento, Ciência e Tecnologia (SEDECT) do Estado do Pará.

Dentre as diversas áreas trabalhadas nesse projeto, existe o subprojeto de Engenharia de Software, que tem como órgão executor o Laboratório de Engenharia de Software (LABES) da UFPA. Esse subprojeto tem como objetivo avançar na institucionalização de padrões arquiteturais e na metodologia de desenvolvimento de software adotada na PRODEPA, visando abrangência e benefícios a toda a administração pública do Estado como um todo.

Nesse sentido, uma pesquisa de campo foi realizada em 2008 tendo como objetivo coletar informações sobre as metodologias de desenvolvimento de software utilizadas e os padrões arquiteturais aplicados no projeto de software em diversos órgãos públicos do Estado (PRODEPA, Seduc, Detran, entre outros). Após a análise dos dados dessa pesquisa, uma das melhorias que deveriam ser implantadas no processo de desenvolvimento de software da PRODEPA estava nas práticas realizadas na fase de testes. Não existia uma padronização a ser seguida durante a execução das atividades de teste e a importância dada a fase de testes (em termos de esforço e custo) era muito baixa se comparada com as demais (análise, projeto e construção).

Dessa forma, um processo de implantação de melhoria de processos foi proposto por membros do LABES (um bolsista com formação em Ciência da Computação em nível de graduação e outros três com pós-graduação e habilitados como implementadores oficiais do MPS.BR) em parceria com a responsável pelo processo de Garantia de Qualidade da PRODEPA (uma funcionária com pós-graduação em Ciência da Computação e habilitada como implementadora MPS.BR), visando estabelecer uma metodologia sistemática de testes que combinasse boas práticas de planejamento e execução de testes com as necessidades e particularidades da PRODEPA. 
Esse processo de implantação de melhoria seguiu as seguintes etapas: Diagnóstico, realizado a partir de entrevistas com a equipe de desenvolvimento da PRODEPA e da análise de artefatos de testes de projetos passados, com o objetivo de encontrar as deficiências na execução dos testes realizados e conhecer as necessidades (tipos de testes aplicáveis) e o ambiente de desenvolvimento (recursos humanos, materiais e ferramentas); Elaboração do Processo de Testes, em que houve a definição do processo padrão de testes da PRODEPA pela equipe do LABES, sendo esse processo validado com a alta diretoria e com o grupo de garantia da qualidade da PRODEPA; Execução e Refinamento, que se deu a partir da introdução do processo definido em projetos pilotos, nos quais houve o acompanhamento (mentoria e avaliação de produtos desenvolvidos) por parte da equipe do LABES e a coleta de métricas, ambos utilizados para refinamento do processo; e Institucionalização do Processo, que vem seguindo uma abordagem em tempo, ou seja, implementado de projeto em projeto de acordo com a demanda da PRODEPA; além da elaboração e divulgação no setor de desenvolvimento de um catálogo de apoio aos testes, contendo: a descrição do processo padrão de testes, orientações para elaboração de casos de testes, e padronização de uso de ferramentas e desenvolvimento de artefatos (casos de teste, bug report, etc).

A seguir será descrito o processo padrão de testes implantado na PRODEPA em termos de atividades, artefatos produzidos, papéis envolvidos, ferramentas e demais componentes que viabilizam a sua execução.

\subsection{Processo de Testes da PRODEPA}

Antes de detalhar o fluxo de trabalho do processo de testes, é necessário descrever a infra-estrutura necessária para desempenhá-lo. Dessa forma, nos itens abaixo serão apresentados os papéis envolvidos, os artefatos produzidos e as ferramentas utilizadas na execução do processo.

\subsubsection{Papéis}

Um funcionário pode assumir diversos papéis funcionais durante o ciclo de vida do processo de testes. Entretanto, devem ser seguidas as seguintes políticas organizacionais:

"O funcionário que assume o papel funcional para desenvolver os casos de teste não deve assumir o papel funcional que desenvolve os produtos a serem testados."

"O funcionário que assume o papel funcional para desenvolver casos de teste não deve assumir o papel funcional que executa os testes para averiguar sua operacionalidade."

Todos os papéis funcionais e suas respectivas responsabilidades dentro do processo de testes da PRODEPA estão descritos na Tabela 1.

Tabela 1 - Papéis Funcionais e Responsabilidades

\begin{tabular}{|l|l|}
\hline Papel Funcional & Responsabilidades \\
\hline Analista de Testes & $\begin{array}{l}\text { 1. Identificar e, posteriormente, definir os Casos de Testes necessários. } \\
\text { 2. Monitorar a abrangência dos testes e avaliar a qualidade geral obtida ao } \\
\text { testar os casos de teste. Deve focar seu trabalho nas técnicas de teste } \\
\text { adequadas ao tipo de teste desenvolvido. }\end{array}$ \\
\hline Gerente de Testes & $\begin{array}{l}\text { 1. Assegurar o planejamento e o gerenciamento apropriados dos recursos } \\
\text { de teste. }\end{array}$ \\
$\begin{array}{l}\text { 2. Avaliar o andamento e a eficácia do esforço de teste. } \\
\text { 3. Averiguar o nível apropriado de qualidade mediante a correção de } \\
\text { defeitos importantes; }\end{array}$ \\
\hline
\end{tabular}




\begin{tabular}{|l|l|}
\hline Projetista de Testes & $\begin{array}{l}\text { 1. Levantar as necessidades relacionadas à montagem da infra-estrutura } \\
\text { de teste, incluindo: ambiente de teste, arquitetura de solução, restrições } \\
\text { tecnológicas, e ferramentas de teste. } \\
\text { 2. Liderar o Processo de Teste no âmbito tecnológico, sobretudo, no uso } \\
\text { de ferramentas }\end{array}$ \\
\hline Testador & $\begin{array}{l}\text { 1. Executar os testes levando em consideração as condições, passos e } \\
\text { cenários documentados no Caso de Teste. }\end{array}$ \\
\hline
\end{tabular}

\subsubsection{Artefatos}

Os produtos de trabalho inerentes do processo de testes servem de entrada e/ou saída para as atividades de teste. Tais artefatos são descritos na Tabela 2.

Tabela 2 - Artefatos do Processo de Teste

\begin{tabular}{|l|l|}
\hline Artefato & Descrição \\
\hline $\begin{array}{l}\text { Plano de } \\
\text { Testes }\end{array}$ & $\begin{array}{l}\text { Descreve a abordagem sistemática utilizada durante o processo de Testes. Neste } \\
\text { documento são definidos: tipo de teste, técnicas utilizadas, funcionários } \\
\text { envolvidos (com seus respectivos papéis funcionais) e a lista de Casos de Teste a } \\
\text { ser trabalhada. }\end{array}$ \\
\hline $\begin{array}{l}\text { Caso de } \\
\text { Testes }\end{array}$ & $\begin{array}{l}\text { Conjunto de entradas, condições de execução e resultados esperados para o teste. } \\
\text { Cada caso de teste é identificado com a finalidade de avaliar um determinado } \\
\text { aspecto do software (Requisitos, Casos de Uso, Regra de Negócio, Componentes } \\
\text { de Software, etc.) }\end{array}$ \\
\hline $\begin{array}{l}\text { Relatório } \\
\text { de Testes }\end{array}$ & $\begin{array}{l}\text { Registra uma visão geral dos resultados dos testes realizados nas atividades } \\
\text { "Executar Testes" e "Corrigir Software". No relato deve constar: } \\
\text { • Variações: Registrar as possíveis variações dos testes realizados em } \\
\text { relação ao planejado na especificação, justificando o motivo de tais variações. } \\
\text { - Abrangência: Avalia se a abrangência do escopo foi suficiente, } \\
\text { conforme o planejado. Devem-se indicar possíveis deficiências nos testes, caso } \\
\text { existam. } \\
\text { • Número de Defeitos: Número total de defeitos encontrados. } \\
\text { • Aprovações: Deve-se se indicar se o item testado foi considerado }\end{array}$ \\
$\begin{array}{l}\text { aprovado ou não (Aprovado | Reprovado) de acordo com um limite de erros } \\
\text { aceitáveis. }\end{array}$
\end{tabular}

\subsubsection{Ferramentas}

As ferramentas utilizadas para desempenhar o processo de testes da PRODEPA auxiliam todos os papéis envolvidos. Tais ferramentas são descritas na Tabela 3.

Tabela 3 - Ferramentas do Processo de Teste

\begin{tabular}{|l|l|}
\hline Ferramenta & Descrição \\
\hline Bugzilla & $\begin{array}{l}\text { Ferramenta de software livre de bug tracking utilizada para monitorar os } \\
\text { defeitos encontrados nos sistemas desenvolvidos. Foi adotada pela empresa } \\
\text { para o gerenciamento dos bugs encontrados durante a execução dos testes. }\end{array}$ \\
\hline $\begin{array}{l}\text { Enterprise } \\
\text { Architect }\end{array}$ & $\begin{array}{l}\text { Ferramenta de suporte à modelagem de sistemas usada para a construção e } \\
\text { armazenamento dos artefatos de documentação. Por disponibilizar uma área } \\
\text { específica para o registro de teste, essa ferramenta foi utilizada para a escrita } \\
\text { dos casos de teste, assim como para o seu resultado. }\end{array}$ \\
\hline Selenium IDE & $\begin{array}{l}\text { Ferramenta para o desenvolvimento e a execução de testes de } \\
\text { funcionalidades dos sistemas. }\end{array}$ \\
\hline DotProject & $\begin{array}{l}\text { Sistema de gerência de projetos utilizado para facilitar a gerenciamento e o } \\
\text { monitoramento das tarefas referentes ao processo de desenvolvimento de } \\
\text { software. }\end{array}$ \\
\hline
\end{tabular}




\subsubsection{Atividades}

Todas as atividades do processo de testes devem ser realizadas por um papel funcional definido, e produzir os artefatos necessários de acordo com as instruções (roteiro) para execução das tarefas.

Um conjunto de atividades padrão para executar os testes foi definido no processo, como pode ser visto na Figura 1. Essas atividades devem ser executadas para cada tipo de teste (Unidade, Integração e Sistema) que se deseje aplicar. Portanto, em um projeto podem ser executados três ciclos de testes: (a) o teste de unidade se aplica a um conjunto de componentes da arquitetura do sistema; (b) o teste de integração se aplica aos casos de uso do sistema; e, (c) o teste de sistema se aplica aos requisitos nãofuncionais (segurança, desempenho, usabilidade, etc.) do sistema.

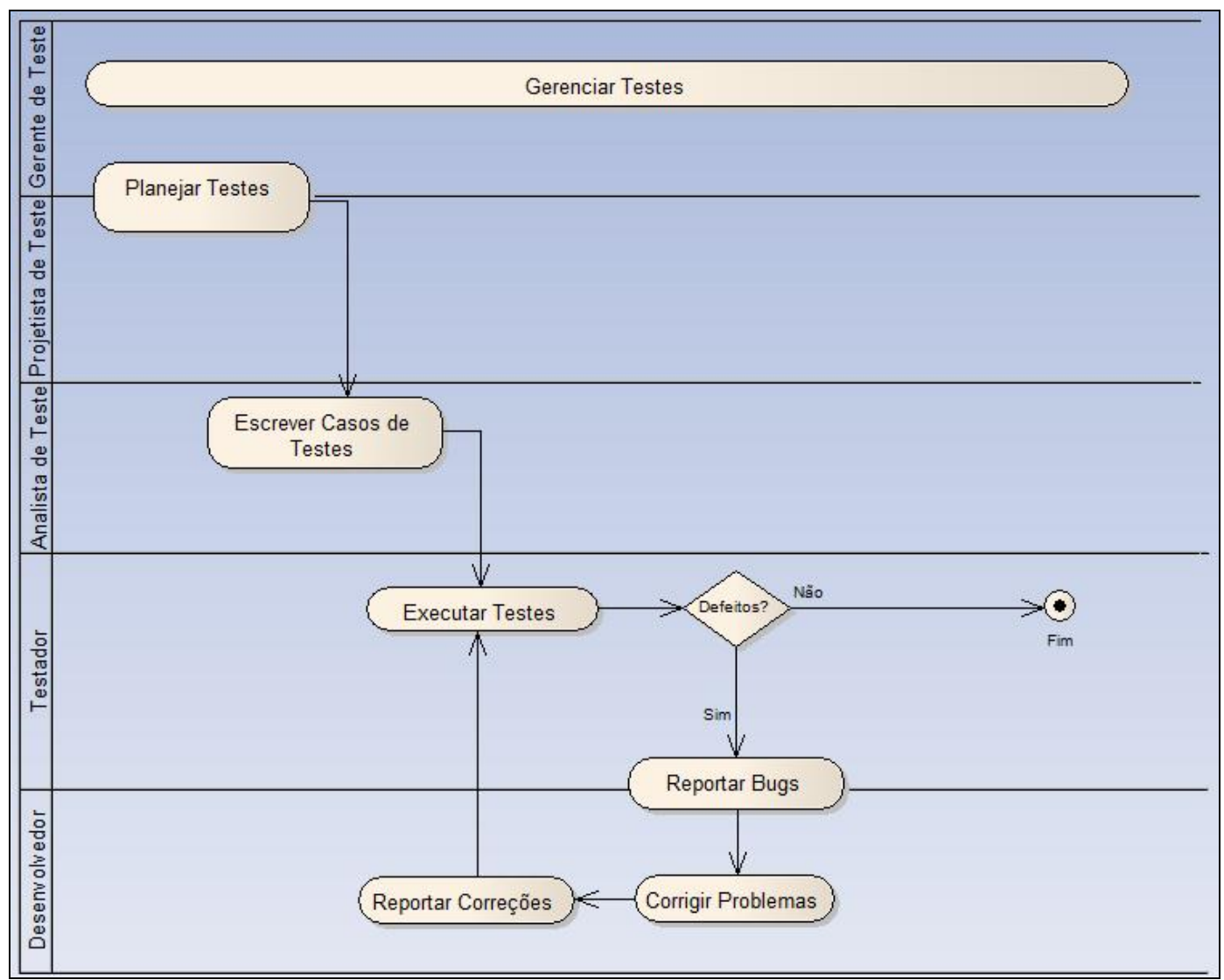

Figura 1 - Fluxo de Atividades do Processo de Testes da PRODEPA

Por fim, a relação entre as atividades, papéis, artefatos e ferramentas do processo de testes é mostrada na Tabela 4.

Tabela 4 - Visão Geral do Processo de Testes

\begin{tabular}{|c|c|c|c|}
\hline Atividades & Papéis/Cargos & Artefatos & Ferramentas \\
\hline Gerenciar Testes & Gerente de Testes & $\begin{array}{c}\text { Relatório de } \\
\text { Testes }\end{array}$ & $\begin{array}{c}\text { DotProject, Enterprise } \\
\text { Architect, Bugzilla }\end{array}$ \\
\hline Planejar Testes & $\begin{array}{c}\text { Gerente de Testes } \\
\text { Projetista de Testes }\end{array}$ & Plano de Testes & DotProject \\
\hline Escrever Casos de & Analista de Testes & Casos de Teste & Enterprise Architect \\
\hline
\end{tabular}




\begin{tabular}{|c|c|c|c|}
\hline Teste & & & \\
\hline $\begin{array}{c}\text { Executar Testes / } \\
\text { Reportar Bugs }\end{array}$ & Testador & Bug Report & $\begin{array}{c}\text { SeleniumIDE, Enterprise } \\
\text { Architect, Bugzilla }\end{array}$ \\
\hline $\begin{array}{c}\text { Corrigir Testes / } \\
\text { Reportar Correções }\end{array}$ & Desenvolvedor & Bug Report & Bugzilla \\
\hline
\end{tabular}

\section{Avaliação Quantitativa do Processo}

Assim como qualquer outra disciplina de engenharia, o desenvolvimento de software requer um mecanismo de medição para obter as informações sobre a execução do processo necessárias para o seu correto entendimento e avaliação [Basili 1994]. Para tal, podem-se utilizar as métricas de software orientadas ao tamanho que são medidas diretas do software e do processo por meio do qual ele é desenvolvido [Pressman 2006].

Nessa categoria, a medida direta mais difundida é a de contagem de linhas de código (LOC). Esse tipo de medida é baseado como o próprio nome sugere na contagem das linhas de código do programa em KLOC (mil linhas de código). Com posse desse dado, é possível calcular a produtividade, qualidade, custo e documentação [Cordeiro 2000] conforme a tabela 3:

Tabela 3 - Métricas orientadas a tamanho

\begin{tabular}{|c|c|c|c|}
\hline Produtividade & Qualidade & Custo & Documentação \\
\hline KLOC/ mês/pessoa & Bugs/KLOC & \$/KLOC & $\begin{array}{c}\text { Página de } \\
\text { documentação/KLOC }\end{array}$ \\
\hline
\end{tabular}

A métrica de qualidade representa a densidade de bugs encontrados nos testes de determinada iteração, através da contabilização de bugs/KLOC registrados. Essa métrica permite avaliar a efetividade dos testes, e prover visibilidade ao gerente de projeto quanto à qualidade e confiabilidade do produto gerado. Se a densidade dos bugs, ou seja, o número de bugs/KLOC estiver aumentando a cada teste de iteração, esse dado nos mostra que o número de bugs inseridos durante as iterações está crescendo, o que ocasionará um aumento de esforço para ajustes do código. Com dados históricos dessa métrica, pode-se estimar o custo e esforço necessário para correção no teste de cada iteração.

$\mathrm{Na}$ PRODEPA, os dados de qualidade foram coletados com o apoio de ferramentas de coleta de métricas automatizadas, e ocorreu em duas etapas. A primeira foi referente à contagem de linhas de código através da utilização da ferramenta Metrics [Metrics 2010], e a segunda refere-se aos números de bugs registrados na ferramenta Bugzilla [Bugzilla 2010].

Para a avaliação do processo, foi observado e analisado o comportamento de projetos que não utilizaram nenhum processo formalizado de teste e o comportamento de projetos que levaram em consideração o processo de teste supracitado.

\subsection{Resultados Obtidos}

Um dos principais resultados da implantação do processo de teste foi o conhecimento adquirido pela equipe de teste, além da visão mais crítica na execução e avaliação dos sistemas para um maior controle de defeitos. Entretanto, realizou-se uma análise quantitativa para ter mais segurança na avaliação do processo de testes. 
A implantação do processo de testes, até o presente momento, se deu em dois projetos críticos da empresa e forneceu os dados iniciais para compor a base histórica de métricas relativas a testes. Após a coleta desses dados (Tabelas 4 e 5) foi possível realizar uma análise das práticas de teste utilizadas, apresentada na Figura 2, a partir da comparação da média da taxa de qualidade em projetos antes e depois da implantação do processo de testes padrão da PRODEPA. Essa análise indica um aumento na qualidade dos projetos no âmbito de achados de bugs.

Tabela 4 - Projetos sem processo institucionalizado de teste

\begin{tabular}{|l|c|c|c|}
\hline Projetos & KLOC & No $^{\text {de Bugs }}$ & Qualidade \\
\hline Projeto 1 & 8,354 & 15 & 1,79554 \\
\hline Projeto 2 & 24,882 & 4 & 0,16075 \\
\hline
\end{tabular}

Tabela 5 - Projetos com processo de teste institucionalizado

\begin{tabular}{|l|c|c|c|}
\hline Projetos & KLOC & No de Bugs $^{\text {Qualidade }}$ \\
\hline Projeto 3 & 71,771 & 253 & 3,52510 \\
\hline Projeto 4 & 30,047 & 36 & 1,19812 \\
\hline
\end{tabular}

É de suma importância destacar os seguintes aspectos sobre os projetos selecionados: todos são do mesmo domínio de aplicações (Gestão Pública), utilizaram a mesma composição de recursos humanos (1 líder de projeto, 1 analista de requisitos, 1 projetista, 2 desenvolvedores e 2 testadores), tiveram o software desenvolvido com o uso das mesmas tecnologias (Hibernate/JPA, Java, EJB3, JBoss, JSF) e, especificamente nos projetos que utilizaram o processo de testes definido, os casos de testes foram avaliados pela equipe de implementação do processo durante $o$ planejamento dos testes. Dessa forma, pode-se minimizar a influência de algumas variáveis (como qualidade dos casos de testes, equipe e software desenvolvido) na avaliação do processo de testes.

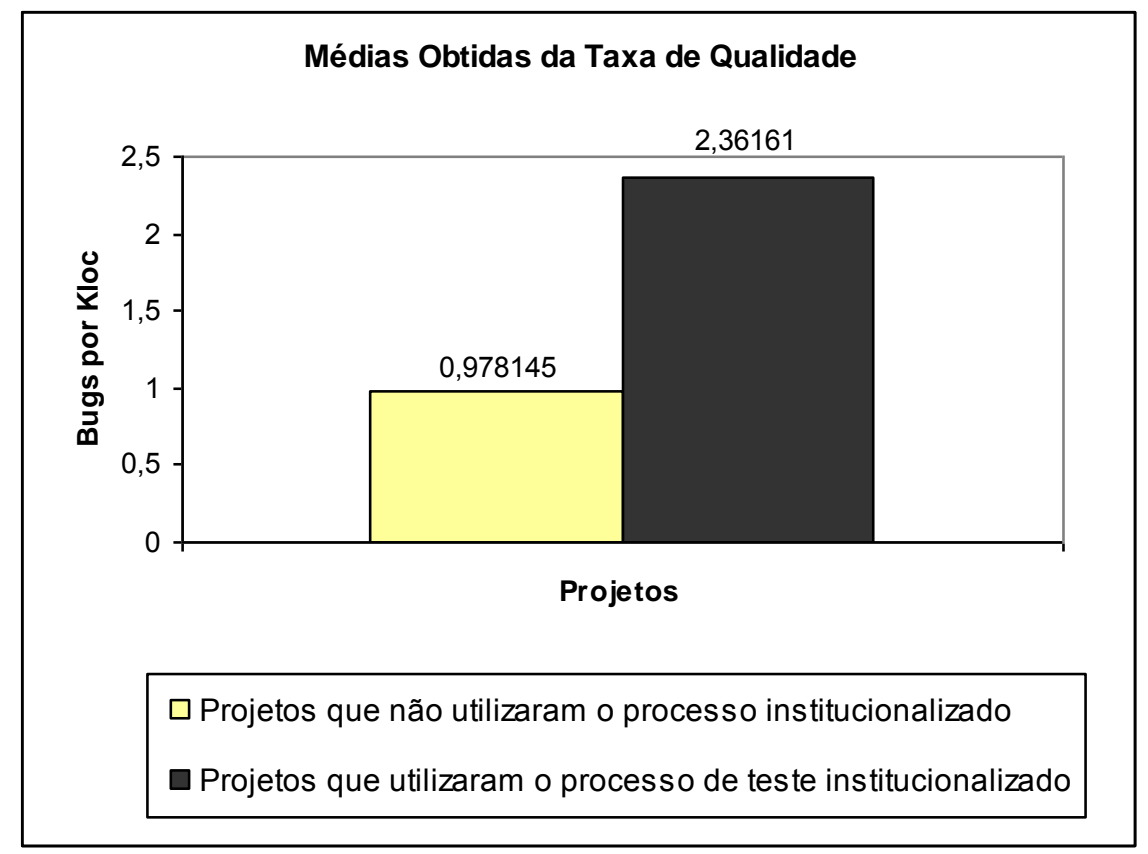

Figura 2 - Médias obtidas da Taxa de Qualidade 


\section{Lições Aprendidas}

Algumas lições aprendidas foram observadas durante a definição e implantação do processo de teste e são relatadas para colaborar com outras empresas que pretendem implantar um processo semelhante. São elas: (i) os artefatos de entrada para a produção dos casos de testes devem ser bem definidos e mantidos; (ii) os casos de teste devem ter relação com o registro gerado na ferramenta de gestão de bugs, para manter a consistência; (iii) os cenários desenvolvidos baseados em telas do sistema auxiliam na confecção dos casos de teste; (iv) a elaboração dos casos de teste deve ser realizada antes da finalização do desenvolvimento, agilizando o processo de testes; (v) a elaboração do projeto de interfaces facilita a construção dos casos de teste; (vi) a comunicação entre os membros do projeto deve ser mantida durante todo o ciclo de vida do projeto, principalmente quando houver modificações; e, (vii) a transferência do conhecimento através da parceria com a academia resultou na eficácia do processo.

\section{Considerações Finais}

Com a implantação do processo de teste, observou-se um resultado preliminar de evolução das atividades de teste, assim como a maturidade da equipe em buscar, através da execução dos casos de teste, uma maior taxa de detecção de defeitos.

As métricas de qualidade nos remeteram a um ganho de achados de bugs, quando comparado com o período anterior a utilização do processo institucionalizado. Assim, a implantação do processo de teste gerou melhorias, tanto no produto entregue para o cliente quanto nos artefatos utilizados na elaboração do projeto.

Mesmo com a aplicação, inicialmente, em dois projetos, o processo mostrou-se eficaz no seu propósito e com isso, a PRODEPA busca ampliar a utilização do processo a todos os projetos de desenvolvimento, além da criação de indicadores que retratem com maior precisão o índice de defeitos, direcionando a uma tomada de decisão para evitar que defeitos cheguem ao cliente.

\section{Referências}

BASILI, V.R., CALDIERA G., ROMBACH H.D. (1994) "Goal Question Metric Paradigm", Encyclopedia of Software Engineering, 2 Volume Set, John Wiley \& Sons, Inc.

BOEHM, B., BASILI, V. (2001) "Software Defect Reduction Top 10 List", IEEE Computer, v. 34, n.1, pp. 135-137.

BUGZILLA. (2010) “Home Bugzilla”, http://www.bugzilla.org/, Fevereiro.

CORDEIRO, Marco Aurélio. (2000) "Métricas de Software", http://www.pr.gov.br/batebyte/edicoes/2000/bb101/metricas.htm, Fevereiro.

CRESPO, Adalberto Nobiato; BUENO, Paulo Marcos Siqueira; SALVIANO, Clenio F.; JINO, Mario. (2008) "Analysis of an Artifact Oriented Test Process Model and of Testing Aspects of ISO/IEC 15504”. In: VII Jornadas Iberoamericanas de Ingeniería de Software e Ingeniería del Conocimiento (JIISIC). Guayaquil, Ecuador, January 30 - February 1, 2008:147-154.

METRICS. (2010) “Metrics 1.3.6”, http://metrics.sourceforge.net/, Fevereiro.

MPS.BR (2010) "Mps.Br - Melhoria de Processos do Software Brasileiro", http://www.softex.br/mpsBr/, Fevereiro.

PRESSMAN, Roger. (2006) “Engenharia de Software”. São Paulo: McGrawHill. 